\title{
Interfacing Biology with Nanoelectronics
}

\section{Peng Chen*}

Division of Bioengineering, School of Chemical \& Biomedical Engineering, Nanyang Technological University, 70 Nanyang Drive, Block N1.3, Singapore

\section{Editorial}

Developing new techniques to detect biomolecules and cell functions is the constant and critical driving force to the advance of biology and medicine. For instances, the invention of glucose sensor in 1962 has dramatically changed the landscape of diagnosis, monitoring, and management of diabetes; the invention of patch-clamp technique in early 1980's (1991 Nobel Prize in physiology and medicine) has revolutionized the study on ion channels and been used as the golden standard to examine potential drugs targeting on these membrane proteins.

Biological processes are spatiotemporally mediated by the dynamic actions of molecular machineries which are often organized in nanoscale domains and exist in extremely low abundance. Therefore, there is a pressing need to develop biosensors that can offer high enough sensitivity to detect trace amount of target molecules or minute biological signals, and high enough spatial and temporal resolution to resolve the subtle biological activities. The emerging nanoelectronic biosensors provide unprecedented possibilities towards such ambition which is unattainable by the conventional bioanalytical methods.

The active conducting channel (sensing element) of a nanoelectronic biosensor is made of nanostructured semiconducting (NS-SC) materials whose current flow is completely or largely confined on the surface, and therefore, highly sensitive to minute electrical perturbations imposed by interacting biomolecules or electrogenic biological activities at or near its surface. Because of their nanoscale dimension, NS-SC materials can intimately interact with, thus highly and locally responsive to, similarly-sized biomolecules or biomolecular machineries. Compared to the conventional methodologies (e.g., biochemical, optical, biophysical), nanoelectronic biosensing may offer readily, rapid, and label-free detection with high or even ultimate (single-molecule) sensitivity, high temporal resolution (sub-millisecond), high spatial resolution (nanoscale), and easy integration with electronic readouts. Using lithographic micro fabrication or advanced nanofabrication techniques, nanoelectronic biosensors can be miniaturized for lab-ona-chip developments and made in array format for parallel detection of multiple analytes.

A number of NS-SC materials can be employed for nanoelectronic biosensing, such as, silicon nanowires (SiNWs) [1], single-walled carbon nanotubes (SWCNTs) [2], and recently discovered graphene and its derivatives [3]. Nanoelectronic sensors provide a versatile platform for a dazzling spectrum of biosensing targets and purposes because the availability of different sensing materials enables various sensing mechanisms and device configurations, and diverse chemistry for biofunctionalization. Each NS-SC material possesses unique advantages and limitations while being applied to a particular application. The choice should be made based on balanced consideration on specific requirements on the performance, detection mechanisms to be utilized, ease of fabrication and recording, cost, and so on.

Nanoelectronic sensors have been used to detect the presence of biomolecules, cells, or microbes at extremely low concentration. Remarkably, SiNW transistors have demonstrated the ability to detect single virus [4]; and single molecule detection of DNA-hybridization kinetics has been achieved by SWCNT transistors [5]. The promising and unique potential of nanoelectronic sensors for molecular diagnosis or studies are clearly evidenced by many studies in the past two decades.

Recently, several groups have attempted to interface nanoelectronic devices with live cells for detection of dynamic cellular activities, such as, secretion of signaling molecules and generation of bioelectrical signals [6,7]. In their landmark work published in 2006, Charles Lieber's group of Harvard University demonstrated the use of array of nanowire transistors for spatially resolved detection, stimulation or inhibition of nerve impulses (electrical spike or action potential produced by ion channel activities in a neuron) and their propagation along individual neurites (slender projection from the cell body of a neuron) [8]. In contrast to the invasive intracellular recording by patch-clamp technique, this nanowire approach provides noninvasive extracellular measurement; as compared to the extracellular recording by metal microelectrode, the nanowire approach offers unraveled spatial resolution. Later, the same group made a nano-transistor device at the tip of a bent silicon nanowire, and used it, as a novel alternative to patch-clamp yet with minimum invasiveness, to intracellularly record action potentials [9]. Very recently, Lieber group demonstrated a new type of SiNW transistor with a branched hollow nanotube to establish connection with the intracellular space for the recording of action potentials [10]. Nanoelectronic sensors can probe at single cell or sub cellular level (even nano-domains). With an array of them, signal distribution and propagation in cell networks could be resolved, e.g., in a brain slice or heart tissue. It is envisaged that nanoelectronicscell interface would be instrumental or revolutionary to the study of cell functions as well as to the discovery of drugs targeting on these functions [11].

Exciting progress has been made in interfacing nanoelectronics with biomolecules, cells, and tissues/organs. No doubt that the convergence of nanoelectronics and biological systems will bring profound impacts on fundamental biological studies, diagnosis, drug discovery, and development of human-machine interface. But currently, nanoelectronic sensing still remains as a scientist niche. To transform it to practical and widely accessible techniques, tremendous and interdisciplinary efforts are still needed, for examples, to develop facile and scalable approach to make these fine devices, and to understand

*Corresponding author: Peng Chen, Division of Bioengineering, School of Chemical \& Biomedical Engineering, Nanyang Technological University, 70 Nanyang Drive, Block N1.3, Singapore, 637457, Tel: +65-6514-1086; E-mail: chenpeng@ntu.edu.sg

Received March 19, 2012; Accepted March 20, 2012; Published March 22, 2012

Citation: Chen P (2012) Interfacing Biology with Nanoelectronics. J Biosens Bioelectron 3:e105. doi:10.4172/2155-6210.1000e105

Copyright: (c) 2012 Chen P. This is an open-access article distributed under the terms of the Creative Commons Attribution License, which permits unrestricted use, distribution, and reproduction in any medium, provided the original author and source are credited. 
better and optimally engineer the interaction/communication between the bio-system and the nanodevice.

\section{References}

1. Chen KI, Li BR, Chen YT (2011) Silicon nanowire field-effect transistor-based biosensors for biomedical diagnosis and cellular recording investigation. Nano Today 6: 131-154.

2. Allen BL, Kichambare PD, Star A (2007) Carbon nanotube field-effect-transistorbased biosensors. Advanced Materials 19: 1439-1451.

3. Liu Y, Dong X, Chen P (2012) Biological and chemical sensors based on graphene materials. Chem Soc Rev 41: 2283-2307.

4. Patolsky F, Zheng G, Hayden O, Lakadamyali M, Zhuang X, et al. (2004) Electrical detection of single viruses. Proc Natl Acad Sci U S A 101: 1401714022

5. Sorgenfrei S, Chiu CY, Gonzalez RL Jr, Yu YJ, Kim P, et al. (2011) Label-free single-molecule detection of DNA-hybridization kinetics with a carbon nanotube field-effect transistor. Nat Nanotechnol 6: 126-132.
6. Huang Y, Cai D, Chen P (2011) Micro and Nanotechnologies for Study of Cell Secretion. Anal Chem 83: 4393-4406.

7. Huang $Y$, Chen $P$ (2010) Nanoelectronic biosensing of dynamic cellular activities based on nanostructured materials. Adv Mater 22: 2818-2823.

8. Patolsky F, Timko BP, Yu G, Fang Y, Greytak AB, et al. (2006) Detection, stimulation, and inhibition of neuronal signals with high-density nanowire transistor arrays. Science 313: 1100-1104.

9. Tian B, Cohen-karni T, Qing Q, Duan X, Xie P, et al. (2010) Three-dimensional, flexible nanoscale field-effect transistors as localized bioprobes. Science 329 830-834.

10. Duan X, Gao R, Xie P, Cohen-karni T, Qing Q, et al. (2011) Intracellular recordings of action potentials by an extracellular nanoscale field-effect transistor. Nat Nanotechnol 7: 174-179.

11. Pui TS, Sudibya HG, Luan X, Zhang Q, Ye F, et al. (2010) Non-invasive detection of cellular bioelectricity based on carbon nanotube devices for highthroughput drug screening. Adv Mater 22: 3199-3203. 